\title{
Research on Arch Effect between Anti-slide Piles and Soil Pressure on Pile Sheet
}

\author{
Li Guo and Xianglan Li
}

Nanchang Key Laboratory of Material and Structure Detection, Jiangxi University of Technology

Keywords: Anti-slide Pile; Arch Effect; Pile Sheet Wall; Soil Pressure; Double-row Pile; Model Test; Row Spacing

\begin{abstract}
At present, the anti-slide piles are widely applied in the field of geotechnical engineering such as the railway, highway, hydropower, municipal and mining engineering due to its advantages of small masonry, large anti-slide resistance, good retaining effect, and so on. With the extensive application of the anti-slide piles, the design ideal and computation theory have been continuously developed. However, a common consensus has not been reached due to the complexity of the rock and soil properties, the arching mechanism of the anti-slide pile, the calculation of soil pressure on the holding sheet, and the retaining effect of large landslide with double-row piles. For this purpose, deep analysis was made in this paper on the distribution law of the soil pressure on the pile sheet and the force transmission mechanism of the double-row piles based on the analysis of the arching effect of civil engineering by taking following research subjects as the basis, including "Research on Three-dimensional Soil Arch Effect of Cantilever Anti-slide Piles", a subject supported by the National Natural Science Foundation of China, and "Demonstration of Optimization Technology Integration for Regional Spatial Planning and Land Use of The Villages and Towns", a subject under the "National Key Technology Development and Research Program during 12th Five-year Plan". On the basis of the above-mentioned study of the soil arch effect between the piles and the soil press ure on the pile sheet, a long-time observation for the soil pressure on the pile sheet was carried out and deep study was made on the creep effect of the soil arch between the piles and the time effect of the soil pressure on the pile sheet by further utilizing the large outdoor test and indoor model test.
\end{abstract}

\section{Introduction}

Anti-slide pile is a cylindrical member for withstanding the landslide thrust, soil resistance and other horizontal forces mainly cylindrical member, the cross section of the pile often use s large cross section of reinforced concrete. It buries the piles in stable formations (slide bed), uses the mutual clamping action of piles and soil around piles, transmits the landslide thrust to the deep stable formations, uses the anchoring and passive forces in the deep formations, and stabilizes the slope.

Anti-slide piles have the following advantages: (1) Compared with the anti-slide retaining wall, it is featured by small masonry, large anti-slide resistance and good retaining effect. Usually a pile can withstand thousands of millions of landslide thrust, and it can also be jointly used in multiple rows of piles, and has good effect of governing large-and-medium sized landslides. (2) Anti-slide pile has small cross-section with small disturbance to the landslide stability. When being supported by reinforced concrete retaining wall, it will not cause large sliding landslide and the construction is safe. (3) When there are many piles, dig holes manually, pour reinforced concrete rectangular or circular 
cross section, the construction might be simultaneously carried out at intervals, and there will be more working surfaces and less interference. During the pile excavation and construction, special mechanical equipment is not needed, which is convenient. (4) Pile position is flexible. According to project needs, set up the piles in the front, middle or rear part of landslides, set them up in a single row or multiple rows, and thus it has greater flexibility.

Based on the extensive application of pile sheet wall and double-row piles and major problems, this paper identifies two main elements: The first is the research on soil arch effect between cantilever piles and the soil pressure on the holding sheet, mainly studies the soil arch effect between the piles and the change rules of retaining plate pressure, deduces the calculation formula for retaining plate soil pressure between the piles; the second is the research on soil arch effect between double rows and the pile thrust between the rows, in order to study the soil arch effect between double rows and the force transmission mechanism between double-row piles, and analyze the reasonable spacing between the double rows and the sliding force in all pile rows on this basis.

This paper comprehensively compares the theory and numerical simulation, and many scholars reach a consensus on the reasonable pile spacing of anti-slide piles by considering the soil arch effect and using the soil between the piles. However, due to lack of experimental verification, the pile spacing is still mainly determined according to engineer experience or analog, and the relevant design specification does not give a clear calculation. In the current study, the main disputes include the arch springing of soil arch between the piles and cross section control.

This paper will combine with the soil arching mechanism analysis between the anti-slide piles, conducts large outdoor thrust test, makes an in-depth study of the soil arch effect between the piles of anti-slide piles and distribution rules of the soil pressure on the pile sheet, analyzes and computes over the soil pressure on the holding sheet between the piles on this basis; based on the single-row pile soil arch effect and transmission mechanism through indoor model thrust test, this paper analyzes the soil arch effect under different row spacing conditions and changes of thrust in different pile rows, puts forward reasonable the calculation method for row spacing of double-row piles and the thrust in different pile rows; finally, remains the thrust unchanged, carries out a long-time observation for the soil pressure on the pile sheet, and studies the influences of soil creep effect on arch effect between the piles and the soil pressure on the pile sheet.

\section{Analysis of Arch Effect between Anti-slide Piles}

Soil arch effect is a common phenomenon in soil mechanics. When excavating tunnel in the soil strata, the soil above the tunnel will deform or move to the tunnel direction, enable the soil above the tunnel to release stress, and deliver to adjacent fixed portion of soil. Typically, the soil above the tunnel will be collapsed, and its upper soil will finally form a stable free surface. From its appearance, the collapsed tunnel top is like an arch.

Soil arch formation is caused by the relative displacement of soil, and the relative displacement process is also an adjustment process of soil stress. Due to the relative displacement modes of different soils, the stress adjustment modes during the arching process also vary. For example, regardless of arch shapes or internal stress distribution forms in the arch, the soil arch effect in the foregoing dam or silt storage silo as well as the soil arch effect in the reinforced retaining wall and box-shaped structure, they are not the same. According to the distribution form of principal stress trajectory after arching, the soil arching effect is divided into minor principal stress arch and major principal stress arch. The analysis of arching mechanism is as follows: 
(1) Minor Principal Stress Arch

(1) Formation Process of Minor Principal Stress Arch

As shown in Fig. 1a, the soil support arch springing is of two parallel vertical wall backs, and assumes the soil is no cohesive soil. In the process of wall soil settlement, being supported by friction between wall soils, the horizontal stress on the wall back is not minor stress, which is incons is tent with Rankine soil pressure without arching effect, and the major stresses at Point A and Point $C$ have been deflected. When the soil between walls reaches the ultimate state, the trajectory of minor principal stress through Point $\mathrm{A}$ and Point $\mathrm{C}$ will form a downward curved arch axis - minor principal stress arch. Minor principal stress arch is different from the load-bearing structural arch, it is a curve representing the trajectory of minor principal stress inside the deformed soil, the minor principal stress arch is vertical downward, and the structural arch projects upward (Fig. 2).

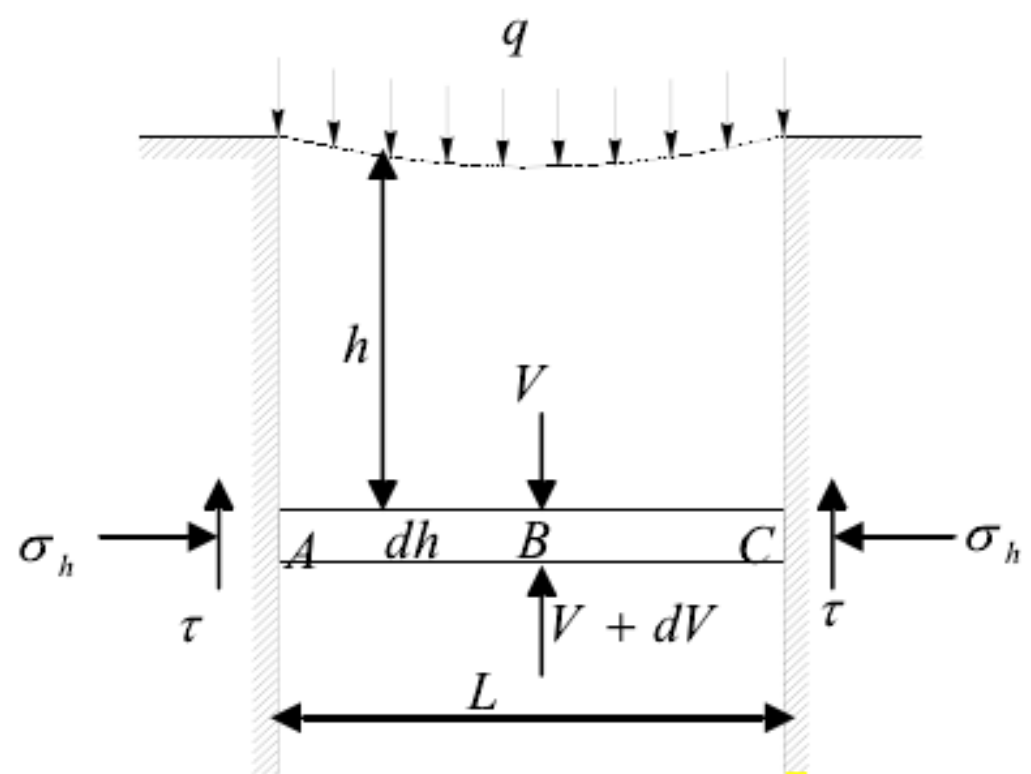

Fig. 1 Analysis of Horizontal Unit Force 


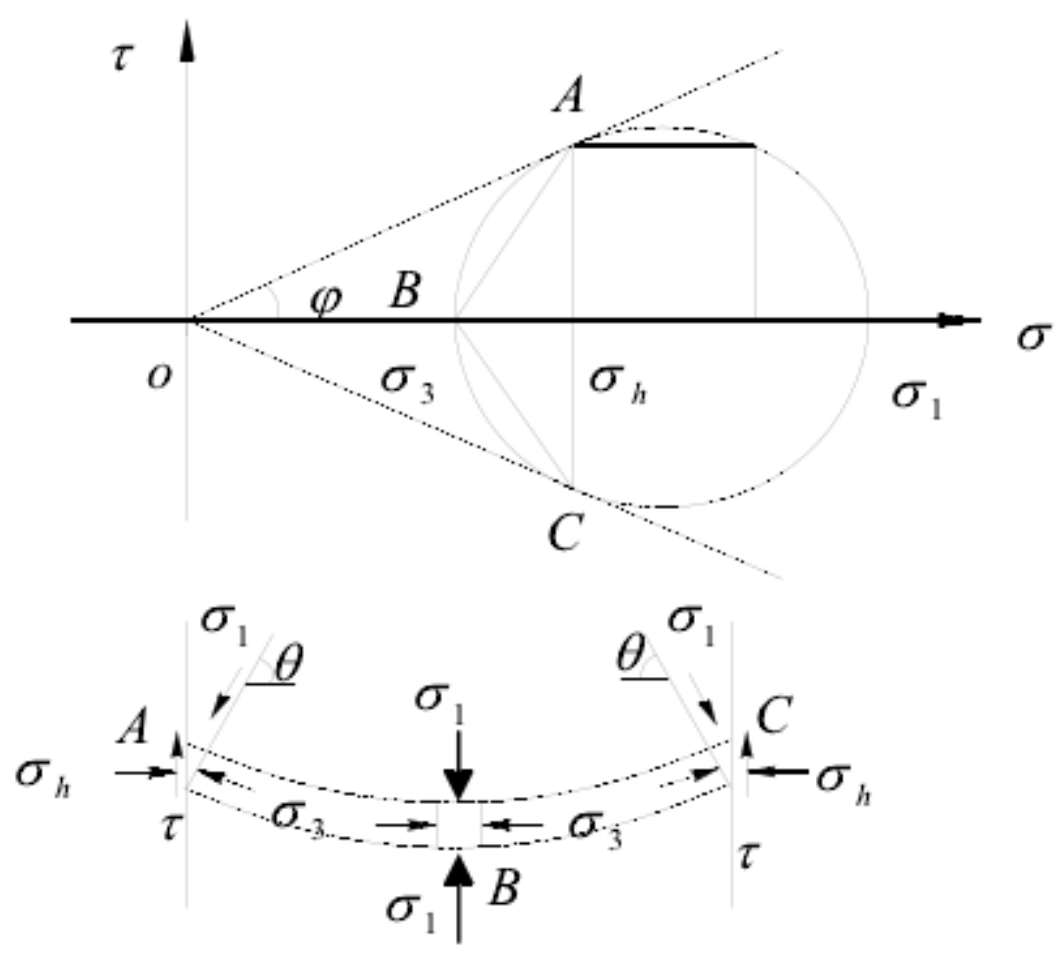

Fig. 2 Analysis of Minor Principle Stress Force

(2) Formation Analysis of Minor Principal Stress Arch

$\mathrm{B}$ is the half arch span length, $\alpha$ is the angle between the major principle stress $\sigma_{1}$ in any differential element of the arch axis and the vertical direction.

Assume $\tan \alpha$ is the linear function of major arch length $\mathrm{S}, \tan \alpha=\mathrm{f}(\mathrm{S})=\mathrm{AS}+\mathrm{C}_{1}$.

When $\mathrm{S}=0, \quad \mathrm{C}_{1}=\tan \alpha$, so $\mathrm{f}(\mathrm{S})=\mathrm{AS}+\tan \alpha_{1}$

\section{Large Thrust Experimental Study on Arch Effect between Single-row Piles}

To prevent collapse and instability of soil between anti-slide piles under the rainfall, eroding or other adverse conditions, it is necessary to set up holding sheet between the anti-slide piles and form the pile sheet retaining wall, which is referred to pile sheet wall. Regardless of application in slope excavation or slope filling, the pile sheet wall transmission mechanism is closed related with the arching effect of soil between the piles, so the pile holding sheet design is more complex than the conventional retaining wall design. It can be found by analyzing the engineering problems and the current calculation method of soil pressure on the holding sheet between the piles. First of all, for the setting of holding sheet between the piles, there are hanging plates before the piles, that is, set up the holding sheet near the pile surface, or set up the holding sheet between the piles at the back side of piles. The arching effect on soil between the piles and transmission of holding sheet setting method remain to be further studied; secondly, the existing calculation assumption of pile sheet wall soil pressure is too simplified. If the value is taken by adopting all soil pressure, the holding sheet design is conservative, and increases the total investment in pile sheet wall project under certain conditions. 
According to the test conditions and similarity criteria, when meeting the geometric similarity, the experiment also takes into main account of physical similarity, stress similarity and load similarity, and thus this paper obtains:

$$
\begin{gathered}
\mathrm{C}_{\sigma}=\mathrm{C}_{\mathrm{c}}=\mathrm{C}_{\varphi}=1 \\
\mathrm{C}_{\mathrm{Z}}=C_{L}=1 / 3
\end{gathered}
$$

Where, $C_{\sigma}$ is the stress similarity coefficient by comparing the model stress with the prototype stress;

$C_{c}$ is the cohesion similarity coefficient by comparing the model sliding cohesion with the prototype sliding cohesion;

$C_{\varphi}$ is the internal friction angle similarity coefficient by comparing the model sliding internal friction angle with the prototype sliding internal friction angle;

$C_{z}$ is the depth similarity coefficient by comparing the model soil depth with the prototype soil depth.

According to the foregoing analysis, the stability of the soil arch between the piles shall consider the impacts of self-weight on arch stability in addition to the impacts of axial force on the arch stability. If the free surface is too high, the arch damage might occur under the gravity stress. Under the phase conditions, with the depth increase, the rational pile spacing also increases. Therefore, in order to accurately analyze the soil arch effect between the piles, the model test shall also consider the impacts of soil weight.

According to the soil gravity stress, this paper respectively has:

$$
\begin{gathered}
\sigma_{\mathrm{m}}=\gamma_{\mathrm{m}} \mathrm{Z}_{\mathrm{m}} \\
\sigma_{\mathrm{p}}=\gamma_{\mathrm{p}} \mathrm{Z}_{\mathrm{p}}
\end{gathered}
$$

Where, $\sigma_{\mathrm{m}}, \gamma_{\mathrm{m}}, \mathrm{Z}_{\mathrm{m}}$ are respectively the gravity stress, medium weight and corresponding depth in the model;

$\sigma_{\mathrm{p}} 、 \gamma_{\mathrm{p}}, \mathrm{Z}_{\mathrm{p}}$ are respectively the gravity stress, medium weight and corresponding depth in the prototype.

The basic concept of similar constant is:

$$
\sigma_{\mathrm{m}}=\gamma_{\sigma} \mathrm{Z}_{\mathrm{p}} \quad \gamma_{\mathrm{m}}=\mathrm{C}_{\mathrm{m}} \gamma_{\mathrm{p}}
$$

\section{Analysis of Arch Effect and Soil Pressure on the Holding Sheet between the piles}

According to the soil arch formation mechanism and the experiment of soil pressure on the pile sheet, when there is arch effect between the piles, under the support of arch, the landslide thrust mainly transmits to the arch springing and arch body through the arch between the piles (Fig. 3), the 
transmission mechanism of soil between the piles or force mode is similar to the bridge and other arch structure. With the thrust load increases, arch axial force of soil between the piles as well as the soil pressure transmitting to the pile back also increases. The holding sheet between the piles mainly undertakes the local soil pressure at the inner side of arch. With the thrust load increase, the soil pressure basically remains unchanged, and the soil pressure on holding sheet is small. The soil damage mainly passes through the inner side of arch, and the arch between the piles and its external side has good overall stability.

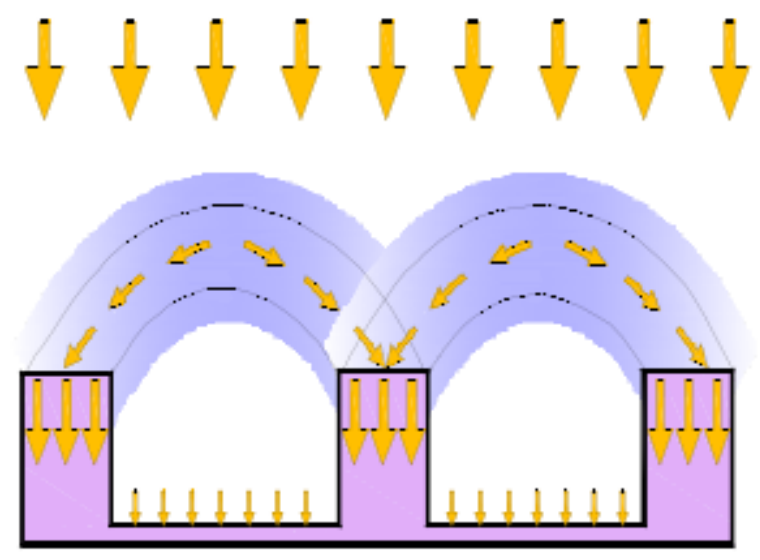

Fig. 3 Arch Effect between the Piles

In contrast, when the arch effect pile develops weakly, the landslide thrust will directly have effect on the soil between the piles and the holding sheet (Fig. 4). With the increase of thrust, the soil pressure effect on the holding sheet increases quickly, and the soil pressure effect on the holding sheet between the piles is large as well. With the holding sheet damage, the soil between the piles will usually have the overall shear failure.

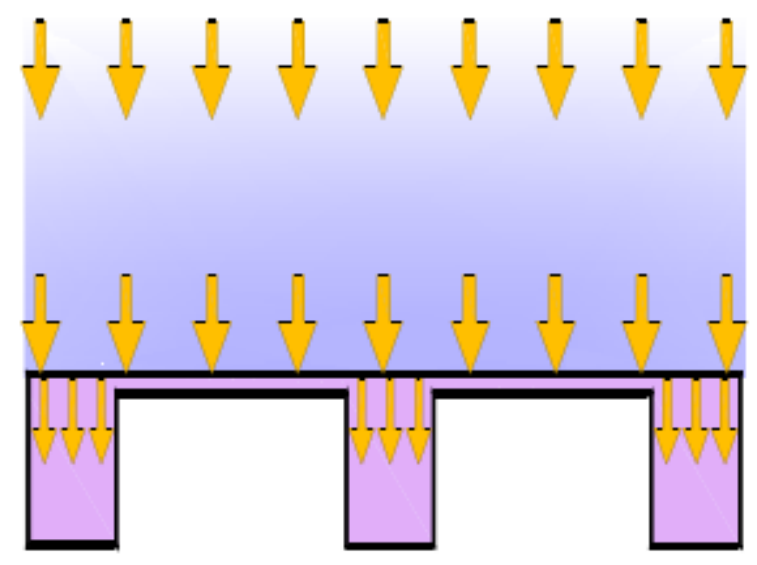

Fig. 4 No Arch Effect between the Piles

In the practical project, on the one hand, the related personnel should make full use of arch effect between the piles, in order to make the soil pressure on the holding sheet between the piles as small as possible, and achieve the purpose of design optimization; on the other hand, when designing holding sheet between the piles, they should take full account of the formation conditions of soil arching between the piles, such as plate setting method and holding sheet stiffness, and determine to 
calculate by local soil pressure (strong arch effect development) or calculate by all soil pressure or landslide thrust (weak arch effect development).

Assumed that the soil pressure transmitting from the arch soil to holding sheet between the piles is evenly distributed in the horizontal direction, and its size is controlled by the vertical pressure, namely:

$$
\sigma_{\mathrm{y}}=\lambda \sigma_{\mathrm{z}}
$$

Where, $\sigma_{\mathrm{y}}$ - soil pressure in the horizontal direction, namely, the soil pressure on the holding sheet,

$\sigma_{\mathrm{z}}$ - pressure in the vertical direction, $\mathrm{kPa}$;

$\lambda$ - coefficient of horizontal pressure in the vertical plane.

Considering the pile cross section height $\mathrm{h}$ is far less than the net distance between the piles, the upward friction resistance effect at the $\sigma_{\mathrm{y}}$ pile side will be omitted.

\section{Conclusions}

Slide pile is used to withstand the cylindrical member of landslide thrust, soil resistance and other horizontal forces. Due to its advantages of small masonry, large anti-slide resistance and good retaining effect, this paper deeply analyzes the additional stress in the soil after the piles based on the soil arching effect analysis, and makes a comprehensive analysis based on the pile spacing factors affecting the stability of arch between the piles; makes an in-depth research on the arch effect between the piles and change rules of the soil pressure on the pile sheet through the large outdoor test, and builds the calculation model for soil pressure on the holding sheet between the piles; on this basis, this paper focuses on deeply studying the soil arch effect between the piles and rows as well as the force transmission rule under different row spacing conditions for the double-row piles to be applied to large and extra large landslides. Through the above research and summary, this paper obtains the following results and understanding.

(1) According to the calculation of soil additional stress caused by arching effect, when the soil is subject to the retaining effect of anti-slide piles in the sliding process, the soil behind the piles will produce the additional stress concentration area in the $\mathrm{x}$ and $\mathrm{y}$ directions, the sliding soil particle will be deflected, produce "wedge-caulking" effect between the piles under certain conditions, and thus form the soil arch. When the pile width increases, the additional stress area in the $\mathrm{x}$ and $\mathrm{y}$ directions will increase accordingly, and vice versa. When changing the load size and soil strength, the additional stress scope in the $\mathrm{x}$ and $\mathrm{y}$ directions remain unchanged.

(2) Based on analyzing the arching mechanism of arch effect between the piles, this paper combines More-Coulomb strength theory with the static equilibrium between the piles, simultaneously considers the geometrical conditions of arch between the piles, and establishes the reasonable pile spacing and arch axis calculation model and formula for the anti-slide piles.

(3) Based on analyzing the soil arch effect between the piles, this paper also analyzes the possibility existing in rock arch between the piles. When the soft rock is retained by anti-slide piles in the downturn, the arch effect will also produce between the anti-slide piles, form the rock arch similar to soil arch, compared with the foregoing soil arch, build the rock arch model, and propose 
the corresponding calculation method, which has initially solve the reasonable pile spacing problems of rock slope anti-sliding retaining.

(4) In order to study the soil arch effect between the piles and the change rule of the soil pressure on the pile sheet, this paper designs the large outdoor thrust test. The test shows that for the plate before the piles, due to thrust load at all levels, obvious soil arch phenomenon exists between the piles. After being obscured by soil arch between the piles, the holding sheet mainly undertakes the soil pressure effect in the local arch region, and the soil pressure effect on holding sheet is relatively small; for the plate behind the piles, the soil arch effect between the piles extremely not obvious.

\section{Acknowledgment}

This work was supported by Project on professional and characteristical construction of Jiangxi province 2010 (Civil Engineering) and Project on the planning and construction of disciplines in Jiangxi University of Technology (Structure Engineering)

\section{References}

[1] Zhou Y, Zhou D P, Feng J. Geometrically mechanical characters of soil arch between two adjacent laterally loaded piles and determination of suitable pile spacing[J]. Yantu Lixue(Rock and Soil Mechanics), 2006, 27(3): 455-457.

[2] Jiang L W, Huang R Q, Jiang Z X. Analysis of soil arching effect between adjacent piles and their spacing in cohesive soils[J]. Yantu Lixue(Rock and Soil Mechanics), 2006, 27(3): 445-450.

[3] Zheng G, Yan Z X, Lei H Y, et al. Field observation and finite element numerical simulation analysis of effect on adjacent piles due to excavation[J]. Yantu Gongcheng Xuebao(Chinese Journal of Geotechnical Engineering), 2007, 29(5): 638-643.

[4] Yang M, Yao L K, Wang G J. Study on effect of width and space of anti-slide piles on soil arching between piles[J]. Yantu Gongcheng Xuebao(Chinese Journal of Geotechnical Engineering), 2007, 29(10): 1477-1482.

[5] Xueqiang Y, Shixiu H, Xinshan Z. On arch effect in civil engineering[J]. Journal of Hubei Polytechnic University, 1994, 9(1).

[6] Chen F, Li A, Lu Y. Improved solution of Hewlett's method for soil arching effect on piled embankments[J]. Yanshilixue Yu Gongcheng Xuebao/Chinese Journal of Rock Mechanics and Engineering, 2007, 26(6): 1278-1283.

[7] Tao L I U X D. A METHOD FOR COMPUTING THE PRESTRESSING FORCE OF THE ANTI-SLIDE PILE WITH PRES TRESSED CABLE [J][J]. Journal of Engineering Geology, 2002, 3: 020 .

[8] Cao W P, Chen R, Chen Y. Experimental investigation on soil arching in piled reinforced embankments[J]. Yantu Gongcheng Xuebao(Chinese Journal of Geotechnical Engineering), 2007, 29(3): 436-441.

[9] Xiao-ming Y E. Calculation of soil pressure on columnpanel structure type retaining wall[J]. Underground Space, 1999, 19(2): 142-146. 
[10] Ying H W, Jiang B, Xie K H. Distribution of active earth pressure against retaining walls considering arching effects $[\mathrm{J}][\mathrm{J}]$. Chinese Journal of Geotechnical Engineering, 2007, 5: 014. 